\title{
The viscosity approximation forward-backward splitting method for the implicit midpoint rule of quasi inclusion problems in Banach spaces
}

\author{
Li Yang*, Fuhai Zhao \\ School of Science, South West University of Science and Technology, Mianyang, Sichuan 621010, China.
}

Communicated by $\mathrm{X}$. Qin

\begin{abstract}
The purpose of this paper is to introduce a viscosity approximation forward-backward splitting method for the implicit midpoint rule of an accretive operators and m-accretive operators in Banach spaces. The strong convergence of this viscosity method is proved under certain assumptions imposed on the sequence of parameters. The results presented in the paper extend and improve some recent results announced in the current literature. Moreover, some applications to the minimization optimization problem and the linear inverse problem are presented. (C)2017 All rights reserved.
\end{abstract}

Keywords: Viscosity approximation, Banach space, splitting method, forward-backward algorithm, the implicit midpoint rule. 2010 MSC: 47H09, 47J25.

\section{Introduction}

Let $X$ be a real Banach space. We study the following inclusion problem: find $x^{*} \in X$ such that

$$
0 \in A x^{*}+B x^{*}
$$

where $A: X \rightarrow X$ is an operator and $B: X \rightarrow 2^{X}$ is a set-valued operator. This problem includes, as special cases, convex programming, variational inequalities, split feasibility problem and minimization problem. To be more precise, some concrete problems in machine learning, image processing and linear inverse problem can be modeled mathematically as this form.

One of the popular iterative methods used for solving problem (1.1) is the forward-backward splitting method $[15,21,27,37]$ which is defined by the following manner: $x_{1} \in X$ and

$$
x_{n+1}=(I+r B)^{-1}\left(x_{n}-r A x_{n}\right), \quad n \geqslant 1,
$$

where $r>0$. We see that each step of iterates involves only with $A$ as the forward step and $B$ as the backward step, but not the sum of $A$ and B. This method includes, in particular, the proximal point

\footnotetext{
*Corresponding author

Email addresses: scmyxkdy1@163.com (Li Yang), scmyxkdzfh@163.com (Fuhai Zhao)
} 
algorithm [7, 9, 19, 24, 33] and the gradient method [6, 18]. Lions-Mercier [21] introduced the following splitting iterative methods in a real Hilbert space:

$$
x_{n+1}=\left(2 J_{r}^{A}-I\right)\left(2 J_{r}^{B}-I\right) x_{n}, \quad n \geqslant 1,
$$

and

$$
x_{n+1}=J_{r}^{A}\left(2 J_{r}^{B}-I\right) x_{n}+\left(I-J_{r}^{B}\right) x_{n}, \quad n \geqslant 1,
$$

where $J_{r}^{\top}=(I+r T)^{-1}$ is the resolvent of $T$. The first one is often called Peaceman-Rachford algorithm [28] and the second one is called Douglas-Rachford algorithm [17]. We note that both algorithms can be weakly convergent in general [27].

In 2012, Takashashi et al. [36] proved some strong convergence theorems of the Halpern-type iteration in a Hilbert space $H$, which is defined by the following manner: for any $x_{1} \in H$,

$$
x_{n+1}=\beta_{n} x_{n}+\left(1-\beta_{n}\right)\left(\alpha_{n} u+\left(1-\alpha_{n}\right) J_{r_{n}}^{B}\left(x_{n}-r_{n} A x_{n}\right)\right), \quad \forall n \geqslant 1,
$$

where $u \in H$ is a given point and $A$ is an $\alpha$-inverse strongly monotone mapping on $H$ and $B$ is a maximal monotone operator on $H,\left\{r_{n}\right\} \subset(0, \infty),\left\{\alpha_{n}\right\},\left\{\beta_{n}\right\} \subset(0,1]$. Under suitable conditions, they proved that the sequence $\left\{x_{n}\right\}$ generated by (1.2) converges strongly to a solution of the inclusion problem (1.1).

Recently, López et al. [22] introduced the following Halpern-type forward-backward method: $x_{1} \in X$ and

$$
x_{n+1}=\alpha_{n} u+\left(1-\alpha_{n}\right)\left(J_{r_{n}}^{B}\left(x_{n}-r_{n}\left(A x_{n}+a_{n}\right)\right)+b_{n}\right) \text {, }
$$

where $u \in X, A$ is an $\alpha$-inverse strongly accretive mapping on $X$ and $B$ is an $m$-accretive operator on $X$. $\left\{r_{n}\right\} \subset(0, \infty),\left\{\alpha_{n}\right\} \subset(0,1]$ and $\left\{a_{n}\right\},\left\{b_{n}\right\}$ are error sequences in $X$. They proved that the sequence $\left\{x_{n}\right\}$ generated by (1.3) strongly converges to a solution of the inclusion problem (1.1) under some appropriate conditions. There have been many works concerning the problem of finding zero points of the sum of two monotone operators (in Hilbert spaces) and accretive operators (in Banach spaces). For more details, see $[11,14,22,29,30,36,37,39,42]$.

In 2015, Cholamjiak [12] studied a generalized forward-backward method for solving the inclusion problem (1.1) for an accretive and m-accretive operators in Banach spaces.

$$
x_{n+1}=\alpha_{n} u+\lambda_{n} x_{n}+\delta_{n} J_{r_{n}}^{B}\left(x_{n}-r_{n} A x_{n}\right)+e_{n}, \quad n \geqslant 1 .
$$

They then proved its strong convergence under some mild conditions.

The viscosity approximation method for nonexpansive mapping in Hilbert spaces was introduced by Moudafi [26], following the ideas of Attouch [2]. Refinements in Hilbert spaces and extensions to Banach spaces were obtained by $\mathrm{Xu}[41]$.

Let $\mathrm{T}: \mathrm{X} \rightarrow \mathrm{X}$ be a nonexpansive mapping and $\mathrm{f}: \mathrm{X} \rightarrow \mathrm{X}$ be a contraction. Explicit viscosity method for nonexpansive mappings generates a sequence $\left\{x_{n}\right\}$ through the iteration process:

$$
x_{n+1}=\alpha_{n} f\left(x_{n}\right)+\left(I-\alpha_{n}\right) T x_{n}, \quad n \geqslant 0,
$$

where $I$ is the identity of $X$. It is well-known $[26,41]$ that under certain conditions, the sequence $\left\{x_{n}\right\}$ converges in norm to a fixed point $q$ of $T$.

The implicit midpoint rule is one of the powerful methods for solving ordinary differential equations, see $[3,4,16,34,35,38]$ and the references therein. For instance, consider the initial value problem for the differential equation $y^{\prime}(t)=f(y(t))$ with the initial condition $y(0)=y_{0}$, where $f$ is a continuous function from $R^{d}$ to $R^{d}$. The implicit midpoint rule is that which generates a sequence $\left\{y_{n}\right\}$ via the relation

$$
\frac{1}{h}\left(y_{n+1}-y_{n}\right)=f\left(\frac{y_{n+1}+y_{n}}{2}\right) \text {. }
$$

The implicit midpoint rule has been extended [1] to nonexpansive mappings, which generates a sequence $\left\{x_{n}\right\}$ by the implicit procedure:

$$
x_{n+1}=\left(1-t_{n}\right) x_{n}+t_{n} T\left(\frac{x_{n+1}+x_{n}}{2}\right), \quad n \geqslant 0 .
$$


Motivated and inspired by the research going on in this direction. The purpose of this paper is to introduce a viscosity approximation forward-backward splitting method for the implicit midpoint rule of an accretive operators and m-accretive operators in the framework of Banach spaces. More precisely, we consider the following iterative algorithm:

$$
x_{n+1}=\alpha_{n} f\left(x_{n}\right)+\lambda_{n} x_{n}+\delta_{n} J_{r_{n}}^{B}\left(I-r_{n} A\right)\left(\frac{x_{n+1}+x_{n}}{2}\right)+e_{n}, \quad n \geqslant 1 .
$$

Under certain assumptions imposed on the sequence of parameters, the strong convergence of this viscosity method is proved. Finally, we discuss applications of algorithms (1.4) to the minimization optimization problem and the linear inverse problem.

\section{2. preliminaries}

In order to prove the main results of the paper, we need the following basic concepts, notations and lemmas.

We assume that $X$ is a real Banach space with norm $\|\cdot\|$ and dual space $X^{*}$. Let $T$ be a nonlinear mapping. We denote the fixed point set of $T$ by $\operatorname{Fix}(T)$.

Let $\delta(\epsilon):(0,2] \rightarrow[0,1]$ be modulus of convexity of $X$ defined by

$$
\delta(\epsilon)=\inf \left\{1-\frac{\|x+y\|}{2}:\|x\|=\|y\|=1,\|x-y\| \geqslant \epsilon\right\} .
$$

A Banach space $X$ is said to be uniformly convex if $\delta(\epsilon)>0$ for every $\epsilon \in(0,2]$.

Let $\rho:[0, \infty) \rightarrow[0, \infty)$ be the modulus of smoothness of $X$ defined by

$$
\rho(t)=\sup \left\{\frac{1}{2}(\|x+t y\|+\|x-t y\|)-1: x, y \in X,\|x\|=\|y\|=1\right\} .
$$

A Banach space $X$ is said to be uniformly smooth if $\frac{\rho(t)}{t} \rightarrow 0$ as $t \rightarrow 0$. Let $q$ be a fixed real number with $q>1$. Then a Banach space $E$ is said to be q-uniformly smooth if there exists a constant $b>0$ such that $\rho(t) \leqslant b t^{q}$ for all $t>0$. It is well-known that every q-uniformly smooth Banach space is uniformly smooth.

Let $\mathrm{J}_{\mathrm{q}}(\mathrm{q}>1)$ denote the generalized duality mapping from $X$ into $2^{\mathrm{X}^{*}}$ given by

$$
\mathrm{J}_{\mathbf{q}}(\mathrm{x})=\left\{\mathrm{j}_{\mathbf{q}}(\mathrm{x}) \in \mathrm{X}^{*}:\left\langle\mathrm{x}, \mathrm{j}_{\mathbf{q}}(\mathrm{x})\right\rangle=\|x\|^{\mathbf{q}},\left\|\mathbf{j}_{\mathbf{q}}(\mathrm{x})\right\|=\|\mathrm{x}\|^{\mathbf{q}-1}\right\}, \quad \forall x \in \mathrm{X},
$$

where $\langle\cdot, \cdot\rangle$ denotes the duality pairing between $X$ and $X^{*}$. In particular, $\mathrm{J}_{2}:=\mathrm{J}$ is called the normalized duality mapping on X. It is also known (e.g., [40, p.1128]]) that

$$
j_{q}(x)=\|x\|^{q-2} J(x), \quad x \neq 0 .
$$

Some properties of the duality mappings are collected as follows.

Lemma 2.1 ([13]). Let $1<\mathrm{q}<\infty$.

(i) The Banach space $\mathrm{X}$ is smooth if and only if the duality mapping $\mathrm{J}_{\mathrm{q}}$ is single-valued.

(ii) The Banach space $\mathrm{X}$ is uniformly smooth if and only if the duality mapping $\mathrm{J}_{\mathrm{q}}$ is single-valued and norm-tonorm uniformly continuous on bounded subsets of X.

Using the concept of sub-differentials, we know the following inequality:

Lemma 2.2 ([10, p. 33]). Let $\mathrm{q}>1$ and $\mathrm{X}$ be a real normed space with the generalized duality mapping $\mathrm{J}_{\mathrm{q}}$. Then, for any $x, y \in X$, we have

$$
\|x+y\|^{q} \leqslant\|x\|^{q}+q\left\langle y, j_{q}(x+y)\right\rangle,
$$

for all $j_{q}(x+y) \in J_{q}(x+y)$. 
Lemma 2.3 ([31, Corollary 1]). Let $\mathrm{C}$ be a closed convex subset of a uniformly smooth Banach space $\mathrm{X}$, and let $\mathrm{T}: \mathrm{C} \rightarrow \mathrm{C}$ be a nonexpansive mapping with a fixed point. Let $\mathrm{x}$ belong to $\mathrm{C}$. Define for each $\mathrm{t} \in(0,1)$, the unique fixed point $x_{\mathrm{t}} \in \mathrm{C}$ of the contraction $\mathrm{C} \ni \mathrm{x}_{\mathrm{t}} \mapsto \mathrm{t} x+(1-\mathrm{t}) \mathrm{T} \mathrm{x}_{\mathrm{t}}$ converges strongly as $\mathrm{t} \rightarrow 0$ to a fixed point of $\mathrm{T}$.

Lemma 2.4 ([23, Lemma 3.1]). Let $\left\{a_{n}\right\}$ and $\left\{\eta_{n}\right\}$ be sequences of nonnegative real numbers such that

$$
a_{n+1} \leqslant\left(1-\gamma_{n}\right) a_{n}+\tau_{n}+\eta_{n}, \quad n \geqslant 1,
$$

where $\left\{\gamma_{n}\right\}$ is a sequence in $(0,1)$ and $\left\{\tau_{n}\right\}$ is a real sequence. Assume $\sum_{n=1}^{\infty} \eta_{n}<\infty$. Then the following results hold:

(i) If $\tau_{n} \leqslant \gamma_{n} M$, for some $M \geqslant 0$, then $\left\{a_{n}\right\}$ is a bounded sequence.

(ii) If $\sum_{n=1}^{\infty} \gamma_{n}=\infty$ and $\limsup _{n \rightarrow \infty} \frac{\tau_{n}}{\gamma_{n}} \leqslant 0$, then $\lim _{n \rightarrow \infty} a_{n}=0$.

Lemma 2.5 ([20, Lemma 8]). Assume $\left\{\mathbf{s}_{\mathrm{n}}\right\}$ is a sequence of nonnegative real numbers such that

$$
s_{n+1} \leqslant\left(1-\gamma_{n}\right) s_{n}+\gamma_{n} \tau_{n}, \quad n \geqslant 1,
$$

and

$$
s_{n+1} \leqslant s_{n}-\eta_{n}+\rho_{n}, \quad n \geqslant 1,
$$

where $\left\{\gamma_{n}\right\}$ is a sequence in $(0,1),\left\{\eta_{n}\right\}$ is a sequence of nonnegative real numbers and $\left\{\tau_{n}\right\}$, and $\left\{\rho_{n}\right\}$ are real sequences such that

(i) $\sum_{n=1}^{\infty} \gamma_{n}=\infty$.

(ii) $\lim _{n \rightarrow \infty} \rho_{n}=0$.

(iii) $\lim _{k \rightarrow \infty} \eta_{n_{k}}=0$ implies $\limsup _{k \rightarrow \infty} \tau_{n_{k}} \leqslant 0$ for any subsequence $\left\{n_{k}\right\} \subset\{n\}$.

Then $\lim _{n \rightarrow \infty} s_{n}=0$.

Lemma 2.6 ([25, p. 63]). Let $\mathrm{q}>1$. Then the following inequality holds:

$$
a b \leqslant \frac{1}{q} a^{q}+\frac{q-1}{q} b^{\frac{q}{q-1}}
$$

for arbitrary positive real numbers $\mathrm{a}$ and $\mathrm{b}$.

Lemma 2.7 ([12, Proposition 3.1]). Let $\mathrm{q}>1$ and let $\mathrm{X}$ be a real smooth Banach space with the generalized duality mapping $j_{q}$. Let $m \in \mathcal{N}$ be fixed. Let $\left\{x_{i}\right\}_{i=1}^{m} \subset X$ and $t_{i} \geqslant 0$ for all $i=1,2, \cdots, m$ with $\sum_{i=1}^{m} t_{i} \leqslant 1$. Then we have

$$
\left\|\sum_{i=1}^{m} t_{i} x_{i}\right\|^{q} \leqslant \frac{\sum_{i=1}^{m} t_{i}\left\|x_{i}\right\|^{q}}{q-(q-1) \sum_{i=1}^{m} t_{i}} .
$$

We define the domain and the range of an operator $A: X \rightarrow 2^{X}$ by $D(A)=\{x \in X: A x \neq \emptyset\}$ and $R(A)=\bigcup\{A z: z \in D(A)\}$, respectively. The inverse of $A$, denoted by $A^{-1}$, is defined by $x \in A^{-1} y$ if 
and only if $y \in A x$. A set-valued operator $A$ is said to be accretive, if for each $x, y \in D(A)$, there exists $j(x-y) \in J(x-y)$ such that

$$
\langle u-v, j(x-y)\rangle \geqslant 0, \quad u \in A x, \quad v \in A y .
$$

An accretive operator $A$ is said to be $m$-accretive if $R(I+r A)=X$, for all $r>0$.

Given $\alpha>0$ and $q \in(1, \infty)$, we say that an accretive operator $A$ is $\alpha$-inverse strongly accretive $(\alpha$-isa) of order $q$, if for each $x, y \in D(A)$, there exists $j_{q}(x-y) \in J(x-y)$ such that

$$
\left\langle u-v, j_{q}(x-y)\right\rangle \geqslant \alpha\|u-v\|^{q}, \quad u \in A x, \quad v \in A y .
$$

In what follows, we shall use the following notation:

$$
\mathrm{T}_{\mathrm{r}}^{\mathrm{A}, \mathrm{B}}=\mathrm{J}_{\mathrm{r}}^{\mathrm{B}}(\mathrm{I}-\mathrm{rA})=(\mathrm{I}+\mathrm{rB})^{-1}(\mathrm{I}-\mathrm{rA}), \quad \mathrm{r}>0 .
$$

Lemma 2.8 ([22, Lemma 3.1 and Lemma 3.2]). Let $X$ be a Banach space. Let $A: X \rightarrow X$ be an $\alpha$-isa of order $\mathrm{q}$ and $\mathrm{B}: \mathrm{X} \rightarrow 2^{\mathrm{X}}$ an m-accretive operator. Then we have

(i) For $r>0, \operatorname{Fix}\left(T_{r}^{A}, B\right)=(A+B)^{-1}(0)$.

(ii) For $0<\mathrm{s} \leqslant \mathrm{r}$ and $\mathrm{x} \in \mathrm{X},\left\|\mathrm{x}-\mathrm{T}_{\mathrm{s}}^{\mathrm{A}, \mathrm{B}} \mathrm{x}\right\| \leqslant 2\left\|\mathrm{x}-\mathrm{T}_{\mathrm{r}}^{\mathrm{A}, \mathrm{B}} \mathrm{x}\right\|$.

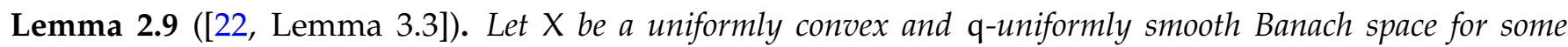
$\mathrm{q} \in(1,2]$. Assume that $\mathrm{A}$ is a single-valued $\alpha$-isa of order $\mathrm{q}$ in $\mathrm{X}$. Then, for given $\mathrm{r}>0$, there exists a continuous, strictly increasing and convex function $\phi_{\mathrm{q}}: \mathrm{R}^{+} \rightarrow \mathrm{R}^{+}$with $\phi_{\mathrm{q}}(0)=0$ such that, for all $\mathrm{x}, \mathrm{y} \in \mathrm{B}_{\mathrm{r}}$,

$$
\begin{aligned}
\left\|T_{r}^{A, B} x-T_{r}^{A, B} y\right\|^{q} \leqslant & \|x-y\|^{q}-r\left(\alpha q-r^{q-1} k_{q}\right)\|A x-A y\|^{q} \\
& -\phi_{q}\left(\left\|\left(I-J_{r}^{B}\right)(I-r A) x-\left(I-J_{r}^{B}\right)(I-r A) y\right\|\right),
\end{aligned}
$$

where $\mathrm{k}_{\mathrm{q}}$ is the $\mathrm{q}$-uniform smoothness coefficient of $\mathrm{X}$.

\section{Main results}

To complete our proof, we need the following proposition:

Proposition 3.1. Let $X$ be a uniformly convex and $q$-uniformly smooth Banach space. Let $A: X \rightarrow X$ be an $\alpha$-isa of order $\mathrm{q}$ and $\mathrm{B}: \mathrm{X} \rightarrow 2^{\mathrm{X}}$ an m-accretive operator such that $\Omega:=(\mathrm{A}+\mathrm{B})^{-1}(0) \neq \emptyset$. Let $\left\{e_{\mathrm{n}}\right\}$ be a sequence in $\mathrm{X}$ and $\mathrm{f}$ be a contraction on $\mathrm{X}$ with coefficient $\beta \in[0,1)$. Let $\left\{x_{n}\right\}$ be generated by $x_{1} \in X$ and

$$
x_{n+1}=\alpha_{n} f\left(x_{n}\right)+\lambda_{n} x_{n}+\delta_{n} J_{r_{n}}^{B}\left(I-r_{n} A\right)\left(\frac{x_{n+1}+x_{n}}{2}\right)+e_{n}, \quad n \geqslant 1,
$$

where $\mathrm{J}_{\mathrm{r}_{n}}^{\mathrm{B}}=\left(\mathrm{I}+\mathrm{r}_{\mathrm{n}} \mathrm{B}\right)^{-1}, 0<\mathrm{r}_{\mathrm{n}}<\left(\alpha \mathrm{q} / \mathrm{k}_{\mathrm{q}}\right)^{1 /(\mathrm{q}-1)}$ and $\left\{\alpha_{n}\right\},\left\{\lambda_{n}\right\}$, and $\left\{\delta_{n}\right\}$ are sequences in $[0,1]$ with $\alpha_{n}+\lambda_{n}+\delta_{n}=1$. If $\sum_{n=1}^{\infty}\left\|e_{n}\right\|<\infty$ or $\lim _{n \rightarrow \infty}\left\|e_{n}\right\| / \alpha_{n}=0$, then $\left\{x_{n}\right\}$ is bounded.

Proof. For each $n \in \mathcal{N}$, we put $T_{n}=J_{r_{n}}^{B}\left(I-r_{n} A\right)$ and let $\left\{y_{n}\right\}$ be defined by

$$
y_{n+1}=\alpha_{n} f\left(y_{n}\right)+\lambda_{n} y_{n}+\delta_{n} T_{n}\left(\frac{y_{n+1}+y_{n}}{2}\right) \text {. }
$$

Firstly, we compute the following:

$$
\begin{aligned}
\left\|x_{n+1}-y_{n+1}\right\|= & \| \alpha_{n}\left(f\left(x_{n}\right)-f\left(y_{n}\right)\right)+\lambda_{n}\left(x_{n}-y_{n}\right) \\
& +\delta_{n}\left(T_{n}\left(\frac{x_{n+1}+x_{n}}{2}\right)-T_{n}\left(\frac{y_{n+1}+y_{n}}{2}\right)\right)+e_{n} \|, \\
\leqslant & \alpha_{n}\left\|f\left(x_{n}\right)-f\left(y_{n}\right)\right\|+\lambda_{n}\left\|x_{n}-y_{n}\right\| \\
& +\delta_{n}\left\|T_{n}\left(\frac{x_{n+1}+x_{n}}{2}\right)-T_{n}\left(\frac{y_{n+1}+y_{n}}{2}\right)\right\|+\left\|e_{n}\right\| \\
\leqslant & \alpha_{n} \beta\left\|x_{n}-y_{n}\right\|+\lambda_{n}\left\|x_{n}-y_{n}\right\| \\
& +\frac{1}{2} \delta_{n}\left(\left\|x_{n}-y_{n}\right\|+\left\|x_{n+1}-y_{n+1}\right\|\right)+\left\|e_{n}\right\| .
\end{aligned}
$$


After simplifying, it follows that

$$
\left(1-\frac{1}{2} \delta_{n}\right)\left\|x_{n+1}-y_{n+1}\right\| \leqslant\left(\alpha_{n} \beta+\lambda_{n}+\frac{1}{2} \delta_{n}\right)\left\|x_{n}-y_{n}\right\|+\left\|e_{n}\right\|
$$

Therefore

$$
\begin{aligned}
\left\|x_{n+1}-y_{n+1}\right\| & \leqslant \frac{2 \alpha_{n} \beta+2 \lambda_{n}+\delta_{n}}{2-\delta_{n}}\left\|x_{n}-y_{n}\right\|+\frac{2}{2-\delta_{n}}\left\|e_{n}\right\| \\
& =\left(1-\frac{2 \alpha_{n}(1-\beta)}{2-\delta_{n}}\right)\left\|x_{n}-y_{n}\right\|+\frac{2}{2-\delta_{n}}\left\|e_{n}\right\| .
\end{aligned}
$$

By the assumptions and Lemma 2.4 (ii), we conclude that $\lim _{n \rightarrow \infty}\left\|x_{n}-y_{n}\right\|=0$. Let $p \in \operatorname{Fix}\left(T_{n}\right)$. We next show that $\left\{y_{n}\right\}$ is bounded. Indeed

$$
\begin{aligned}
\left\|y_{n+1}-p\right\|= & \left\|\alpha_{n}\left(f\left(y_{n}\right)-p\right)+\lambda_{n}\left(y_{n}-p\right)+\delta_{n}\left(T_{n}\left(\frac{y_{n+1}+y_{n}}{2}\right)-p\right)\right\| \\
\leqslant & \alpha_{n}\left\|f\left(y_{n}\right)-p\right\|+\lambda_{n}\left\|y_{n}-p\right\|+\delta_{n}\left\|T_{n}\left(\frac{y_{n+1}+y_{n}}{2}\right)-p\right\| \\
\leqslant & \alpha_{n}\left(\left\|f\left(y_{n}\right)-f(p)\right\|+\|f(p)-p\|\right)+\lambda_{n}\left\|y_{n}-p\right\| \\
& +\frac{1}{2} \delta_{n}\left(\left\|y_{n}-p\right\|+\left\|y_{n+1}-p\right\|\right) \\
\leqslant & \alpha_{n} \beta\left\|y_{n}-p\right\|+\alpha_{n}\|f(p)-p\|+\lambda_{n}\left\|y_{n}-p\right\| \\
& +\frac{1}{2} \delta_{n}\left(\left\|y_{n}-p\right\|+\left\|y_{n+1}-p\right\|\right) .
\end{aligned}
$$

By simplifying, we have

$$
\left(1-\frac{1}{2} \delta_{n}\right)\left\|y_{n+1}-p\right\| \leqslant\left(\alpha_{n} \beta+\lambda_{n}+\frac{1}{2} \delta_{n}\right)\left\|y_{n}-p\right\|+\alpha_{n}\|f(p)-p\|
$$

Hence

$$
\begin{aligned}
\left\|y_{n+1}-p\right\| & \leqslant \frac{2 \alpha_{n} \beta+2 \lambda_{n}+\delta_{n}}{2-\delta_{n}}\left\|y_{n}-p\right\|+\frac{2 \alpha_{n}}{2-\delta_{n}}\|f(p)-p\| \\
& =\left(1-\frac{2 \alpha_{n}(1-\beta)}{2-\delta_{n}}\right)\left\|y_{n}-p\right\|+\frac{2 \alpha_{n}}{2-\delta_{n}}\|f(p)-p\| .
\end{aligned}
$$

This shows that $\left\{y_{n}\right\}$ is bounded by Lemma 2.4 (i) and hence $\left\{x_{n}\right\}$ is also bounded.

We are now ready to prove our main result.

Theorem 3.2. Let $X$ be a uniformly convex and q-uniformly smooth Banach space, $q \in(1,2]$. Let $A: X \rightarrow X$ be an $\alpha$-isa of order $\mathrm{q}$ and $\mathrm{B}: \mathrm{X} \rightarrow 2^{\mathrm{X}}$ an m-accretive operator such that $\Omega:=(\mathrm{A}+\mathrm{B})^{-1}(0) \neq \emptyset$. Let $\left\{e_{\mathrm{n}}\right\}$ be a sequence in $X$ and $f$ be a contraction on $X$ with coefficient $\beta \in[0,1)$. Let $\left\{x_{n}\right\}$ be generated by $x_{1} \in X$ and

$$
x_{n+1}=\alpha_{n} f\left(x_{n}\right)+\lambda_{n} x_{n}+\delta_{n} J_{r_{n}}^{B}\left(I-r_{n} A\right)\left(\frac{x_{n+1}+x_{n}}{2}\right)+e_{n}, \quad n \geqslant 1,
$$

where $\mathrm{J}_{r_{n}}^{\mathrm{B}}=\left(\mathrm{I}+\mathrm{r}_{\mathrm{n}} \mathrm{B}\right)^{-1},\left\{\mathrm{r}_{\mathrm{n}}\right\} \subset(0, \infty)$ and $\left\{\alpha_{n}\right\},\left\{\lambda_{n}\right\}$, and $\left\{\delta_{n}\right\}$ are sequences in $[0,1]$ with $\alpha_{n}+\lambda_{n}+\delta_{n}=1$. Assume that

(i) $\sum_{n=1}^{\infty} \alpha_{n}=\infty, \lim _{n \rightarrow \infty} \alpha_{n}=0$;

(ii) $0<\liminf _{n \rightarrow \infty} r_{n} \leqslant \limsup _{n \rightarrow \infty} r_{n}<\left(\alpha q / k_{q}\right)^{1 /(q-1)}$;

(iii) $\liminf _{n \rightarrow \infty} \delta_{n}>0$; 
(iv) $\sum_{n=1}^{\infty}\left\|e_{n}\right\|<\infty$ or $\lim _{n \rightarrow \infty}\left\|e_{n}\right\| / \alpha_{n}=0$.

Then $\left\{x_{n}\right\}$ strongly converges to some $z \in \Omega$.

Proof. Let $z \in \operatorname{Fix}\left(\mathrm{T}_{\mathrm{n}}\right)$, from Lemma 2.2 and Lemma 2.6, we have

$$
\begin{aligned}
\left\|y_{n+1}-z\right\|^{q}= & \left\|\alpha_{n}\left(f\left(y_{n}\right)-z\right)+\lambda_{n}\left(y_{n}-z\right)+\delta_{n}\left(T_{n}\left(\frac{y_{n+1}+y_{n}}{2}\right)-z\right)\right\|^{q} \\
\leqslant & \left\|\lambda_{n}\left(y_{n}-z\right)+\delta_{n}\left(T_{n}\left(\frac{y_{n+1}+y_{n}}{2}\right)-z\right)\right\|^{q}+q \alpha_{n}\left\langle\left(f\left(y_{n}\right)-z\right), j_{q}\left(y_{n+1}-z\right)\right\rangle \\
\leqslant & \left\|\lambda_{n}\left(y_{n}-z\right)+\delta_{n}\left(T_{n}\left(\frac{y_{n+1}+y_{n}}{2}\right)-z\right)\right\|^{q} \\
& +q \alpha_{n}\left\langle f\left(y_{n}\right)-f(z), j_{q}\left(y_{n+1}-z\right)\right\rangle+q \alpha_{n}\left\langle f(z)-z, j_{q}\left(y_{n+1}-z\right)\right\rangle \\
\leqslant & \left\|\lambda_{n}\left(y_{n}-z\right)+\delta_{n}\left(T_{n}\left(\frac{y_{n+1}+y_{n}}{2}\right)-z\right)\right\|^{q} \\
& +q \alpha_{n} \beta\left\|y_{n}-z\right\|\left\|y_{n+1}-z\right\|^{q-1}+q \alpha_{n}\left\langle f(z)-z, j_{q}\left(y_{n+1}-z\right)\right\rangle \\
\leqslant & \left\|\lambda_{n}\left(y_{n}-z\right)+\delta_{n}\left(T_{n}\left(\frac{y_{n+1}+y_{n}}{2}\right)-z\right)\right\|^{q} \\
& +q \alpha_{n} \beta\left(\frac{1}{q}\left\|y_{n}-z\right\|^{q}+\frac{q-1}{q}\left\|y_{n+1}-z\right\|^{q}\right)+q \alpha_{n}\left\langle f(z)-z, j_{q}\left(y_{n+1}-z\right)\right\rangle \\
\leqslant & \left\|\lambda_{n}\left(y_{n}-z\right)+\delta_{n}\left(T_{n}\left(\frac{y_{n+1}+y_{n}}{2}\right)-z\right)\right\|^{q} \\
& +\alpha_{n} \beta\left\|y_{n}-z\right\|^{q}+(q-1) \alpha_{n} \beta\left\|y_{n+1}-z\right\|^{q}+q \alpha_{n}\left\langle f(z)-z, j_{q}\left(y_{n+1}-z\right)\right\rangle .
\end{aligned}
$$

On the other hand, by Lemma 2.7 and Lemma 2.9, we obtain

$$
\begin{aligned}
\| \lambda_{n}\left(y_{n}-z\right)+\delta_{n} & \left(T_{n}\left(\frac{y_{n+1}+y_{n}}{2}\right)-z\right) \|^{q} \\
\leqslant & \frac{1}{\alpha_{n} q+1-\alpha_{n}}\left(\lambda_{n}\left\|y_{n}-z\right\|^{q}+\delta_{n}\left\|T_{n}\left(\frac{y_{n+1}+y_{n}}{2}\right)-z\right\|^{q}\right) \\
\leqslant & \frac{1}{\alpha_{n} q+1-\alpha_{n}}\left(\lambda_{n}\left\|y_{n}-z\right\|^{q}\right. \\
& +\delta_{n}\left(\left\|\frac{y_{n+1}+y_{n}}{2}-z\right\|^{q}-r_{n}\left(\alpha q-r_{n}^{q-1} k_{q}\right)\left\|A\left(\frac{y_{n+1}+y_{n}}{2}\right)-A z\right\|^{q}\right. \\
& \left.\left.-\phi_{q}\left(\left\|\frac{y_{n+1}+y_{n}}{2}-r_{n} A\left(\frac{y_{n+1}+y_{n}}{2}\right)-T_{n}\left(\frac{y_{n+1}+y_{n}}{2}\right)+r_{n} A z\right\|\right)\right)\right) \\
\leqslant & \frac{1}{\alpha_{n} q+1-\alpha_{n}}\left(\lambda_{n}\left\|y_{n}-z\right\|^{q}\right. \\
& +\delta_{n}\left(\left(\frac{1}{2}\left\|y_{n+1}-z\right\|^{q}+\frac{1}{2}\left\|y_{n}-z\right\|^{q}\right)-r_{n}\left(\alpha q-r_{n}^{q-1} k_{q}\right)\left\|A\left(\frac{y_{n+1}+y_{n}}{2}\right)-A z\right\|^{q}\right. \\
& \left.\left.-\phi_{q}\left(\left\|\frac{y_{n+1}+y_{n}}{2}-r_{n} A\left(\frac{y_{n+1}+y_{n}}{2}\right)-T_{n}\left(\frac{y_{n+1}+y_{n}}{2}\right)+r_{n} A z\right\|\right)\right)\right) \\
\leqslant & \frac{\lambda_{n}+\frac{1}{2} \delta_{n}}{\alpha_{n} q+1-\alpha_{n}}\left\|y_{n}-z\right\|^{q}+\frac{1}{2} \delta_{n} \\
& -\frac{\delta_{n} r_{n}\left(\alpha q-r_{n}^{q}-1 k_{q}\right)}{\alpha_{n} q+1-\alpha_{n}}\left\|A\left(\frac{y_{n+1}+y_{n}}{2}\right)-A z\right\|_{n}^{q} \\
& -\frac{\delta_{n}}{\alpha_{n} q+1-\alpha_{n}} \phi_{q}\left(\left\|\left(\frac{y_{n}+1+y_{n}}{2}\right)-r_{n} A\left(\frac{y_{n+1}+y_{n}}{2}\right)-T_{n}\left(\frac{y_{n+1}+y_{n}}{2}\right)+r_{n} A z\right\|\right) .
\end{aligned}
$$


Replacing (3.3) into (3.2), it follows that

$$
\begin{aligned}
\left\|y_{n+1}-z\right\|^{q} \leqslant & \frac{\lambda_{n}+\frac{1}{2} \delta_{n}}{\alpha_{n} q+1-\alpha_{n}}\left\|y_{n}-z\right\|^{q}+\frac{\frac{1}{2} \delta_{n}}{\alpha_{n} q+1-\alpha_{n}}\left\|y_{n+1}-z\right\|^{q} \\
& -\frac{\delta_{n} r_{n}\left(\alpha q-r_{n}^{q-1} k_{q}\right)}{\alpha_{n} q+1-\alpha_{n}}\left\|A\left(\frac{y_{n+1}+y_{n}}{2}\right)-A z\right\|^{q} \\
& -\frac{\delta_{n}}{\alpha_{n} q+1-\alpha_{n}} \phi_{q}\left(\left\|\left(\frac{y_{n+1}+y_{n}}{2}\right)-r_{n} A\left(\frac{y_{n+1}+y_{n}}{2}\right)-T_{n}\left(\frac{y_{n+1}+y_{n}}{2}\right)+r_{n} A z\right\|\right) \\
& +\alpha_{n} \beta\left\|y_{n}-z\right\|^{q}+(q-1) \alpha_{n} \beta\left\|y_{n+1}-z\right\|^{q}+q \alpha_{n}\left\langle f(z)-z, j_{q}\left(y_{n+1}-z\right)\right\rangle \\
\leqslant & \frac{\lambda_{n}+\frac{1}{2} \delta_{n}+\alpha_{n} \beta\left(\alpha_{n} q+1-\alpha_{n}\right)}{\alpha_{n} q+1-\alpha_{n}}\left\|y_{n}-z\right\|^{q} \\
& +\frac{\frac{1}{2} \delta_{n}+(q-1) \alpha_{n} \beta\left(\alpha_{n} q+1-\alpha_{n}\right)}{\alpha_{n} q+1-\alpha_{n}}\left\|y_{n+1}-z\right\|^{q} \\
& -\frac{\delta_{n} r_{n}\left(\alpha q-r_{n}^{q}-1 k_{q}\right)}{\alpha_{n} q+1-\alpha_{n}}\left\|A\left(\frac{y_{n+1}+y_{n}}{2}\right)-A z\right\|^{q} \\
& -\frac{\delta_{n}}{\alpha_{n} q+1-\alpha_{n}} \phi_{q}\left(\left\|\left(\frac{y_{n+1}+y_{n}}{2}\right)-r_{n} A\left(\frac{y_{n+1}+y_{n}}{2}\right)-T_{n}\left(\frac{y_{n+1}+y_{n}}{2}\right)+r_{n} A z\right\|\right) \\
& +q \alpha_{n}\left\langle f(z)-z, j_{q}\left(y_{n+1}-z\right)\right\rangle .
\end{aligned}
$$

After simplifying it follows that

$$
\begin{aligned}
\left\|y_{n+1}-z\right\|^{q} \leqslant & \left(1-\frac{\alpha_{n} q\left(1-\beta-\beta \alpha_{n}(q-1)\right)}{\left(1-(q-1) \alpha_{n} \beta\right)\left(\alpha_{n} q+1-\alpha_{n}\right)-\frac{1}{2} \delta_{n}}\right)\left\|y_{n}-z\right\|^{q} \\
& -\frac{\delta_{n} r_{n}\left(\alpha q-r_{n}^{q-1} k_{q}\right)}{\left(1-(q-1) \alpha_{n} \beta\right)\left(\alpha_{n} q+1-\alpha_{n}\right)-\frac{1}{2} \delta_{n}}\left\|A\left(\frac{y_{n+1}+y_{n}}{2}\right)-A z\right\|^{q} \\
& -\frac{\delta_{n}}{\left(1-(q-1) \alpha_{n} \beta\right)\left(\alpha_{n} q+1-\alpha_{n}\right)-\frac{1}{2} \delta_{n}} \\
& \times \phi_{q}\left(\left\|\left(\frac{y_{n+1}+y_{n}}{2}\right)-r_{n} A\left(\frac{y_{n+1}+y_{n}}{2}\right)-T_{n}\left(\frac{y_{n+1}+y_{n}}{2}\right)+r_{n} A z\right\|\right) \\
& +\frac{q \alpha_{n}\left(\alpha_{n} q+1-\alpha_{n}\right)}{\left(1-(q-1) \alpha_{n} \beta\right)\left(\alpha_{n} q+1-\alpha_{n}\right)-\frac{1}{2} \delta_{n}}\left\langle f(z)-z, j_{q}\left(y_{n+1}-z\right)\right\rangle .
\end{aligned}
$$

We can check that $\frac{\alpha_{n} q\left(1-\beta-(q-1) \alpha_{n} \beta\right)}{\left(1-(q-1) \alpha_{n} \beta\right)\left(\alpha_{n} q+1-\alpha_{n}\right)-\frac{1}{2} \delta_{n}}$ is in $(0,1)$ since $1<q \leqslant 2,\left\{\alpha_{n}\right\} \subset(0,1)$ and $\lim _{n \rightarrow \infty} \alpha_{n}=0$. Moreover, by condition (ii), $\frac{\delta_{n} r_{n}\left(\alpha q-r_{n}^{q-1} k_{q}\right)}{\left(1-(q-1) \alpha_{n} \beta\right)\left(\alpha_{n} q+1-\alpha_{n}\right)-\frac{1}{2} \delta_{n}}$ and $\frac{\delta_{n}}{\left(1-(q-1) \alpha_{n} \beta\right)\left(\alpha_{n} q+1-\alpha_{n}\right)-\frac{1}{2} \delta_{n}}$ are positive.

For each $n \geqslant 1$, we set

$$
\begin{aligned}
s_{n}= & \left\|y_{n}-z\right\|^{q}, \gamma_{n}=\frac{\alpha_{n} q\left(1-\beta-(q-1) \alpha_{n} \beta\right)}{\left(1-(q-1) \alpha_{n} \beta\right)\left(\alpha_{n} q+1-\alpha_{n}\right)-\frac{1}{2} \delta_{n}} \\
\tau_{n}= & \frac{\alpha_{n} q+1-\alpha_{n}}{1-\beta-(q-1) \alpha_{n} \beta}\left\langle f(z)-z, j_{q}\left(y_{n+1}-z\right)\right\rangle, \\
\eta_{n}= & -\frac{\delta_{n} r_{n}\left(\alpha q-r_{n}^{q-1} k_{q}\right)}{\left(1-(q-1) \alpha_{n} \beta\right)\left(\alpha_{n} q+1-\alpha_{n}\right)-\frac{1}{2} \delta_{n}}\left\|A\left(\frac{y_{n+1}+y_{n}}{2}\right)-A z\right\|^{q} \\
& -\frac{\delta_{n}}{\left(1-(q-1) \alpha_{n} \beta\right)\left(\alpha_{n} q+1-\alpha_{n}\right)-\frac{1}{2} \delta_{n}} \\
& \times \phi_{q}\left(\left\|\left(\frac{y_{n+1}+y_{n}}{2}\right)-r_{n} A\left(\frac{y_{n+1}+y_{n}}{2}\right)-T_{n}\left(\frac{y_{n+1}+y_{n}}{2}\right)+r_{n} A z\right\|\right), \\
\rho_{n}= & \frac{q \alpha_{n}\left(\alpha_{n} q+1-\alpha_{n}\right)}{\left(1-(q-1) \alpha_{n} \beta\right)\left(\alpha_{n} q+1-\alpha_{n}\right)-\frac{1}{2} \delta_{n}}\left\langle f(z)-z, j_{q}\left(y_{n+1}-z\right)\right\rangle .
\end{aligned}
$$


From (3.4), we then have

$$
s_{n+1} \leqslant\left(1-\gamma_{n}\right) s_{n}+\gamma_{n} \tau_{n}, \quad n \geqslant 1,
$$

and

$$
s_{n+1} \leqslant s_{n}-\eta_{n}+\rho_{n}, \quad n \geqslant 1 .
$$

Since $\sum_{n=1}^{\infty} \alpha_{n}=\infty$, it follows that $\sum_{n=1}^{\infty} \gamma_{n}=\infty$. By the boundedness of $\left\{y_{n}\right\}$ and $\lim _{n \rightarrow \infty} \alpha_{n}=0$, we see that $\lim _{n \rightarrow \infty} \rho_{n}=0$. In order to complete the proof, using Lemma 2.5, it remains to show that $\lim _{k \rightarrow \infty} \eta_{n_{k}}=0$ implies $\lim \sup \tau_{n_{k}} \leqslant 0$ for any subsequence $\left\{n_{k}\right\} \subset\{n\}$.

Let $\left\{n_{k}\right\}$ be a subsequence of $\{n\}$ such that $\lim _{k \rightarrow \infty} \eta_{n_{k}}=0$. So, by our assumptions and the property of $\phi_{\mathrm{q}}$, we can deduce that

$$
\begin{aligned}
\lim _{k \rightarrow \infty} & \left\|A\left(\frac{y_{n_{k}+1}+y_{n_{k}}}{2}\right)-A z\right\| \\
= & \lim _{k \rightarrow \infty}\left\|\left(\frac{y_{n_{k}+1}+y_{n_{k}}}{2}\right)-\gamma_{n_{k}} A\left(\frac{y_{n_{k}+1}+y_{n_{k}}}{2}\right)-T_{n_{k}}\left(\frac{y_{n_{k}+1}+y_{n_{k}}}{2}\right)+r_{n_{k}} A z\right\|=0 .
\end{aligned}
$$

This gives, by the triangle inequality, that

$$
\lim _{k \rightarrow \infty}\left\|T_{n_{k}}\left(\frac{y_{n_{k}+1}+y_{n_{k}}}{2}\right)-\frac{y_{n_{k}+1}+y_{n_{k}}}{2}\right\|=0 .
$$

By (3.1), we have

$$
\begin{aligned}
\left\|y_{n_{k}+1}-y_{n_{k}}\right\|= & \left\|\alpha_{n_{k}} f\left(y_{n_{k}}\right)+\lambda_{n_{k}} y_{n_{k}}+\delta_{n_{k}} T_{n_{k}}\left(\frac{y_{n_{k}+1}+y_{n_{k}}}{2}\right)-y_{n_{k}}\right\| \\
\leqslant & \alpha_{n_{k}}\left\|f\left(y_{n_{k}}\right)-y_{n_{k}}\right\|+\delta_{n_{k}}\left\|T_{n_{k}}\left(\frac{y_{n_{k}+1}+y_{n_{k}}}{2}\right)-y_{n_{k}}\right\| \\
\leqslant & \alpha_{n_{k}}\left\|f\left(y_{n_{k}}\right)-y_{n_{k}}\right\|+\delta_{n_{k}}\left\|T_{n_{k}}\left(\frac{y_{n_{k}+1}+y_{n_{k}}}{2}\right)-\frac{y_{n_{k}+1}+y_{n_{k}}}{2}\right\| \\
& +\delta_{n_{k}}\left\|\frac{y_{n_{k}+1}+y_{n_{k}}}{2}-y_{n_{k}}\right\| .
\end{aligned}
$$

By simplifying we have

$$
\left\|y_{n_{k}+1}-y_{n_{k}}\right\| \leqslant \frac{\alpha_{n_{k}}}{1-\frac{1}{2} \delta_{n_{k}}}\left\|f\left(y_{n_{k}}\right)-y_{n_{k}}\right\|+\frac{\delta_{n_{k}}}{1-\frac{1}{2} \delta_{n_{k}}}\left\|T_{n_{k}}\left(\frac{y_{n_{k}+1}+y_{n_{k}}}{2}\right)-\frac{y_{n_{k}+1}+y_{n_{k}}}{2}\right\| .
$$

By Proposition 3.1, $\left\{y_{n}\right\}$ is bounded, and so is $\left\{f\left(x_{n}\right)\right\}$, by condition (i) and (3.5), we obtain

$$
\lim _{k \rightarrow \infty}\left\|y_{n_{k}+1}-y_{n_{k}}\right\|=0 \text {. }
$$

By the triangle inequality, we have

$$
\begin{aligned}
\left\|T_{n_{k}} y_{n_{k}}-y_{n_{k}}\right\| \leqslant & \left\|T_{n_{k}} y_{n_{k}}-T_{n_{k}}\left(\frac{y_{n_{k}+1}+y_{n_{k}}}{2}\right)\right\| \\
& +\left\|T_{n_{k}}\left(\frac{y_{n_{k}+1}+y_{n_{k}}}{2}\right)-\frac{y_{n_{k}+1}+y_{n_{k}}}{2}\right\| \\
& +\left\|\frac{y_{n_{k}+1}+y_{n_{k}}}{2}-y_{n_{k}}\right\| \\
\leqslant & \left\|y_{n_{k}+1}-y_{n_{k}}\right\|+\left\|T_{n_{k}}\left(\frac{y_{n_{k}+1}+y_{n_{k}}}{2}\right)-\frac{y_{n_{k}+1}+y_{n_{k}}}{2}\right\| .
\end{aligned}
$$

By (3.5) and (3.6), we have

$$
\lim _{k \rightarrow \infty}\left\|T_{n_{k}} y_{n_{k}}-y_{n_{k}}\right\|=0 .
$$


Since $\liminf _{k \rightarrow \infty} r_{n}>0$, there is $r>0$ such that $r_{n} \geqslant r$ for all $n \geqslant 1$. In particular, $r_{n_{k}} \geqslant r$ for all $k \geqslant 1$. Lemma 2.8 (ii) yields that

$$
\left\|T_{r}^{A, B} y_{n_{k}}-y_{n_{k}}\right\| \leqslant 2\left\|T_{n_{k}} y_{n_{k}}-y_{n_{k}}\right\|
$$

Then, by (3.7), we obtain

$$
\limsup _{k \rightarrow \infty}\left\|T_{r}^{A, B} y_{n_{k}}-y_{n_{k}}\right\| \leqslant 2 \lim _{k \rightarrow \infty}\left\|T_{n_{k}} y_{n_{k}}-y_{n_{k}}\right\|
$$

It follows that

$$
\lim _{k \rightarrow \infty}\left\|T_{r}^{A, B} y_{n_{k}}-y_{n_{k}}\right\|=0 .
$$

Let $z_{t}=\operatorname{tf}\left(z_{t}\right)+(1-t) T_{r}^{A}, B z_{t}, t \in(0,1)$. Employing Lemma 2.3, we have $z_{t} \rightarrow z \in \Omega$ as $t \rightarrow 0$, from Lemma 2.2 we have that

$$
\begin{aligned}
\left\|z_{t}-y_{n_{k}}\right\|^{\mathrm{q}}= & \left\|t\left(f\left(z_{t}\right)-y_{n_{k}}\right)+(1-t)\left(T_{r}^{A, B} z_{t}-y_{n_{k}}\right)\right\|^{q} \\
\leqslant & (1-t)^{\mathrm{q}}\left\|T_{r}^{A, B} z_{t}-y_{n_{k}}\right\|^{\mathrm{q}}+q t\left\langle f\left(z_{t}\right)-y_{n_{k}}, j_{q}\left(z_{t}-y_{n_{k}}\right)\right\rangle \\
\leqslant & (1-t)^{\mathrm{q}}\left(\left\|T_{r}^{A, B} z_{t}-T_{r}^{A, B} y_{n_{k}}\right\|+\left\|T_{r}^{A, B} y_{n_{k}}-y_{n_{k}}\right\|\right)^{q} \\
& +q t\left\langle f\left(z_{t}\right)-z_{t}, j_{q}\left(z_{t}-y_{n_{k}}\right)\right\rangle+q t\left\langle z_{t}-y_{n_{k}}, j_{q}\left(z_{t}-y_{n_{k}}\right)\right\rangle \\
\leqslant & (1-t)^{q}\left(\left\|z_{t}-y_{n_{k}}\right\|+\left\|T_{r}^{A, B} y_{n_{k}}-y_{n_{k}}\right\|\right)^{q} \\
& +q t\left\langle f\left(z_{t}\right)-z_{t}, j_{q}\left(z_{t}-y_{n_{k}}\right)\right\rangle+q t\left\|z_{t}-y_{n_{k}}\right\|^{q} .
\end{aligned}
$$

This shows that

$$
\left\langle z_{t}-f\left(z_{t}\right), j_{q}\left(z_{t}-y_{n_{k}}\right)\right\rangle \leqslant \frac{(1-t)^{q}}{q t}\left(\left\|z_{t}-y_{n_{k}}\right\|+\left\|T_{r}^{A, B} y_{n_{k}}-y_{n_{k}}\right\|\right)^{q}+\frac{q t-1}{q t}\left\|z_{t}-y_{n_{k}}\right\|^{q} .
$$

From (3.8), we obtain

$$
\begin{aligned}
\limsup _{k \rightarrow \infty}\left\langle z_{t}-f\left(z_{t}\right), j_{q}\left(z_{t}-y_{n_{k}}\right)\right\rangle & \leqslant \frac{(1-t)^{q}}{q t} M^{q}+\frac{q t-1}{q t} M^{q} \\
& =\frac{(1-t)^{q}+q t-1}{q t} M^{q}
\end{aligned}
$$

where $M=\limsup _{k \rightarrow \infty}\left\|z_{t}-y_{n_{k}}\right\|, t \in(0,1)$. We see that $\frac{(1-t)^{q}+q t-1}{q t} \rightarrow 0$ as $t \rightarrow 0$. From Lemma 2.1 (ii), we know that $j_{q}$ is norm-to-norm uniformly continuous on bounded subsets of $X$. Since $z_{t} \rightarrow z$ as $t \rightarrow 0$, we have $\left\|j_{\mathrm{q}}\left(z_{\mathrm{t}}-\mathrm{y}_{\mathrm{n}_{\mathrm{k}}}\right)-\mathrm{j}_{\mathrm{q}}\left(z-\mathrm{y}_{\mathrm{n}_{\mathrm{k}}}\right)\right\| \rightarrow 0$ as $\mathrm{t} \rightarrow 0$. Observe that

$$
\begin{aligned}
& \left|\left\langle z_{\mathrm{t}}-\mathrm{f}\left(z_{\mathrm{t}}\right), j_{\mathrm{q}}\left(z_{\mathrm{t}}-\mathrm{y}_{\mathrm{n}_{\mathrm{k}}}\right)\right\rangle-\left\langle z-\mathrm{f}(z), \mathrm{j}_{\mathrm{q}}\left(z-\mathrm{y}_{\mathrm{n}_{\mathrm{k}}}\right)\right\rangle\right| \\
& \leqslant\left|\left\langle z_{\mathrm{t}}-z+z-\mathrm{f}(z)+\mathrm{f}(z)-\mathrm{f}\left(z_{\mathrm{t}}\right), j_{\mathrm{q}}\left(z_{\mathrm{t}}-\mathrm{y}_{\mathrm{n}_{\mathrm{k}}}\right)\right\rangle-\left\langle z-\mathrm{f}(z), \mathrm{j}_{\mathrm{q}}\left(z-\mathrm{y}_{\mathrm{n}_{\mathrm{k}}}\right)\right\rangle\right| \\
& \leqslant\left|\left\langle z_{\mathrm{t}}-z, j_{\mathrm{q}}\left(z_{\mathrm{t}}-y_{n_{\mathrm{k}}}\right)\right\rangle\right|+\left|\left\langle z-\mathrm{f}(z), j_{\mathrm{q}}\left(z_{\mathrm{t}}-\mathrm{y}_{n_{\mathrm{k}}}\right)-j_{\mathrm{q}}\left(z-y_{n_{\mathrm{k}}}\right)\right\rangle\right| \\
& +\left|\left\langle f(z)-f\left(z_{\mathrm{t}}\right), j_{\mathrm{q}}\left(z_{\mathrm{t}}-y_{n_{\mathrm{k}}}\right)\right\rangle\right| \\
& \leqslant(1+\beta)\left\|z_{\mathfrak{t}}-z\right\|\left\|z_{\mathfrak{t}}-y_{n_{k}}\right\|^{\mathbf{q}-1}+\|z-f(z)\|\left\|j_{\mathbf{q}}\left(z_{\mathfrak{t}}-y_{n_{k}}\right)-j_{\mathbf{q}}\left(z-y_{n_{k}}\right)\right\|^{\mathbf{q}-1} \text {. }
\end{aligned}
$$

So, as $t \rightarrow 0$, we get

$$
\left\langle z_{\mathrm{t}}-\mathrm{f}\left(z_{\mathrm{t}}\right), j_{\mathrm{q}}\left(z_{\mathrm{t}}-\mathrm{y}_{n_{\mathrm{k}}}\right)\right\rangle \rightarrow\left\langle z-\mathrm{f}(z), j_{\mathrm{q}}\left(z-y_{n_{k}}\right)\right\rangle .
$$

From (3.9), as $t \rightarrow 0$, it follows that

$$
\limsup _{k \rightarrow \infty}\left\langle z-f(z), j_{q}\left(z-y_{n_{k}}\right)\right\rangle \leqslant 0 .
$$


Combining (3.6) and (3.10), we get that

$$
\limsup _{k \rightarrow \infty}\left\langle z-f(z), j_{q}\left(z-y_{n_{k}+1}\right)\right\rangle \leqslant 0 .
$$

It also follows that $\limsup _{k \rightarrow \infty} \tau_{n_{k}} \leqslant 0$. We conclude that $\lim _{n \rightarrow \infty} s_{n}=0$ by Lemma 2.5. Hence $y_{n} \rightarrow z$ as $n \rightarrow \infty$, by Proposition 3.1, $\lim _{n \rightarrow \infty}\left\|x_{n}-y_{n}\right\|=0$, so $\lim _{n \rightarrow \infty} x_{n}=z \in \Omega$. We thus complete the proof.

By setting $\lambda_{n}=0$ for all $n \geqslant 1$, we obtain the following result:

Corollary 3.3. Let $X$ be a uniformly convex and $q$-uniformly smooth Banach space, $q \in(1,2]$. Let $A: X \rightarrow X$ be an $\alpha$-isa of order $\mathrm{q}$ and $\mathrm{B}: \mathrm{X} \rightarrow 2^{\mathrm{X}}$ an m-accretive operator such that $\Omega:=(\mathrm{A}+\mathrm{B})^{-1}(0) \neq \emptyset$. Let $\left\{e_{\mathrm{n}}\right\}$ be a sequence in $\mathrm{X}$ and $\mathrm{f}$ be a contraction on $\mathrm{X}$ with coefficient $\beta \in[0,1)$. Let $\left\{\mathrm{x}_{\mathrm{n}}\right\}$ be generated by $\mathrm{x}_{1} \in \mathrm{X}$ and

$$
x_{n+1}=\alpha_{n} f\left(x_{n}\right)+\left(1-\alpha_{n}\right) J_{r_{n}}^{B}\left(I-r_{n} A\right)\left(\frac{x_{n+1}+x_{n}}{2}\right)+e_{n}, \quad n \geqslant 1,
$$

where $\mathrm{J}_{\mathrm{r}_{n}}^{\mathrm{B}}=\left(\mathrm{I}+\mathrm{r}_{\mathrm{n}} \mathrm{B}\right)^{-1},\left\{\mathrm{r}_{n}\right\} \subset(0, \infty)$ and $\left\{\alpha_{n}\right\}$ is a sequences in $[0,1]$. Assume that

(i) $\sum_{n=1}^{\infty} \alpha_{n}=\infty, \lim _{n \rightarrow \infty} \alpha_{n}=0$;

(ii) $0<\liminf _{n \rightarrow \infty} r_{n} \leqslant \limsup _{n \rightarrow \infty} r_{n}<\left(\alpha q / k_{q}\right)^{1 /(q-1)}$;

(iii) $\sum_{n=1}^{\infty}\left\|e_{n}\right\|<\infty$ or $\lim _{n \rightarrow \infty}\left\|e_{n}\right\| / \alpha_{n}=0$.

Then $\left\{x_{n}\right\}$ strongly converges to some $z \in \Omega$.

\section{Applications}

Firstly, we apply Theorem 3.2 to the convex minimization problem. Let $\mathrm{H}$ be a real Hilbert space. Let $\mathrm{F}: \mathrm{H} \rightarrow \mathrm{R}$ be a convex smooth function and $\mathrm{G}: \mathrm{H} \rightarrow \mathrm{R}$ be a convex, lower-semicontinuous and nonsmooth function. We consider the problem of finding $x^{*} \in \mathrm{H}$ such that

$$
F\left(x^{*}\right)+G\left(x^{*}\right) \leqslant F(x)+G(x),
$$

for all $x \in \mathrm{H}$. This problem (4.1) is equivalent, by Fermats rule, to the problem of finding $x^{*} \in \mathrm{H}$ such that

$$
0 \in \nabla \mathrm{F}\left(\mathrm{x}^{*}\right)+\partial \mathrm{G}\left(\mathrm{x}^{*}\right),
$$

where $\nabla F$ is the gradient of $F$ and $\partial G$ is the subdifferential of $G$. In this point of view, we can set $A=\nabla F$ and $B=\partial G$ in Theorem 3.2. This is because if $\nabla F$ is $(1 / L)$-Lipschitz continuous, then it is L-inverse strongly monotone [5, Corollary 10]. Moreover, $\partial \mathrm{G}$ is maximal monotone [32, Theorem A]. So we obtain the following result.

Theorem 4.1. Let $\mathrm{H}$ be real Hilbert space. Let $\mathrm{F}: \mathrm{H} \rightarrow \mathrm{R}$ be a convex and differentiable function with $(1 / \mathrm{L})-$ Lipschitz continuous gradient $\nabla \mathrm{F}$ and $\mathrm{G}: \mathrm{H} \rightarrow \mathrm{R}$ be a convex and lower semi-continuous function which $\mathrm{F}+\mathrm{G}$ attains a minimizer. Let $\left\{e_{\mathrm{n}}\right\}$ be a sequence in $\mathrm{H}$ and $\mathrm{f}$ be a contraction on $\mathrm{X}$ with coefficient $\beta \in[0,1)$. Let $\left\{\mathrm{x}_{\mathrm{n}}\right\}$ be generated by $\mathrm{x}_{1} \in \mathrm{H}$ and

$$
x_{n+1}=\alpha_{n} f\left(x_{n}\right)+\lambda_{n} x_{n}+\delta_{n} J_{r_{n}}\left(I-r_{n} \nabla F\right)\left(\frac{x_{n+1}+x_{n}}{2}\right)+e_{n}, \quad n \geqslant 1,
$$

where $\mathrm{J}_{\mathrm{r}_{n}}=\left(\mathrm{I}+\mathrm{r}_{n} \partial \mathrm{G}\right)^{-1},\left\{\mathrm{r}_{n}\right\} \subset(0, \infty)$ and $\left\{\alpha_{n}\right\},\left\{\lambda_{n}\right\}$, and $\left\{\delta_{n}\right\}$ are sequences in $[0,1]$ with $\alpha_{n}+\lambda_{n}+\delta_{n}=1$. Assume that 
(i) $\sum_{n=1}^{\infty} \alpha_{n}=\infty, \lim _{n \rightarrow \infty} \alpha_{n}=0$;

(ii) $0<\liminf _{n \rightarrow \infty} r_{n} \leqslant \limsup _{n \rightarrow \infty} r_{n}<2 L$;

(iii) $\liminf _{n \rightarrow \infty} \delta_{n}>0$;

(iv) $\sum_{n=1}^{\infty}\left\|e_{n}\right\|<\infty$ or $\lim _{n \rightarrow \infty}\left\|e_{n}\right\| / \alpha_{n}=0$.

Then $\left\{x_{n}\right\}$ strongly converges to a minimizer of $\mathrm{F}+\mathrm{G}$.

Secondly, we apply Theorem 3.2 to solve the unconstrained linear system

$$
\mathrm{C} x=\mathrm{d},
$$

where $C$ is a bounded linear operator on $H$ and $d \in H$. For each $x \in H$, we define $F: H \rightarrow R$ by

$$
\mathrm{F}(\mathrm{x})=\frac{1}{2}\|\mathrm{C} x-\mathrm{d}\|^{2} .
$$

From [8] we know that $\nabla F(x)=C^{\top}(C x-d)$ and $\nabla F$ is $K$-Lipschitz continuous with $K$ the largest eigenvalue of $C^{\top} \mathrm{C}$. So we obtain the following result.

Theorem 4.2. Let $\mathrm{H}$ be real Hilbert space. Let $\mathrm{C}: \mathrm{H} \rightarrow \mathrm{H}$ be a bounded linear operator and $\mathrm{d} \in \mathrm{H}$ with $\mathrm{K}$ the largest eigenvalue of $\mathrm{C}^{\top} \mathrm{C}$. Let $\left\{\mathrm{e}_{\mathrm{n}}\right\}$ be a sequence in $\mathrm{H}$ and $\mathrm{f}$ be a contraction on $\mathrm{X}$ with coefficient $\beta \in[0,1)$. Let $\left\{x_{n}\right\}$ be generated by $x_{1} \in \mathrm{H}$ and

$$
x_{n+1}=\alpha_{n} f\left(x_{n}\right)+\lambda_{n} x_{n}+\delta_{n}\left(I-r_{n} C^{\top}(C-d I)\right)\left(\frac{x_{n+1}+x_{n}}{2}\right)+e_{n}, \quad n \geqslant 1,
$$

where $\left\{r_{n}\right\} \subset(0, \infty)$ and $\left\{\alpha_{n}\right\},\left\{\lambda_{n}\right\}$, and $\left\{\delta_{n}\right\}$ are sequences in $[0,1]$ with $\alpha_{n}+\lambda_{n}+\delta_{n}=1$. Assume that

(i) $\sum_{n=1}^{\infty} \alpha_{n}=\infty, \lim _{n \rightarrow \infty} \alpha_{n}=0$;

(ii) $0<\liminf _{n \rightarrow \infty} r_{n} \leqslant \limsup _{n \rightarrow \infty} r_{n}<2 / K$;

(iii) $\liminf _{n \rightarrow \infty} \delta_{n}>0$;

(iv) $\sum_{n=1}^{\infty}\left\|e_{n}\right\|<\infty$ or $\lim _{n \rightarrow \infty}\left\|e_{n}\right\| / \alpha_{n}=0$.

If (4.2) is consistent, then $\left\{x_{n}\right\}$ strongly converges to a solution of a linear system.

\section{Acknowledgment}

This study was supported by Scientic Reserch Fund of Sichuan Provincial Education Department (No.15ZA0112).

\section{References}

[1] M. A. Alghamdi, N. Shahzad, H.-K. Xu, The implicit midpoint rule for nonexpansive mappings, Fixed Point Theory Appl., 2014 (2014), 9 pages. 1

[2] H. Attouch, Viscosity solutions of minimization problems, SIAM J. Optim., 6 (1996), 769-806. 1

[3] W. Auzinger, R. Frank, Asymptotic error expansions for stiff equations: an analysis for the implicit midpoint and trapezoidal rules in the strongly stiff case, Numer. Math., 56 (1989), 469-499.1 
[4] G. Bader, P. Deuflhard, A semi-implicit mid-point rule for stiff systems of ordinary differential equations, Numer. Math., 41 (1983), 373-398. 1

[5] J. B. Baillon, G. Haddad, Quelques proprits des oprateurs angle-borns et n-cycliquement monotones, (French) Israel J. Math., 26 (1977), 137-150. 4

[6] D. P. Bertsekas, J. N. Tsitsiklis, Parallel and distributed computation: numerical methods, Englewood Cliffs: Prentice Hall, NJ, (1989). 1

[7] H. Brézis, P.-L. Lions, Produits infinis de résolvantes, (French) Israel J. Math., 29 (1978), 329-345. 1

[8] C. Byrne, A unified treatment of some iterative algorithms in signal processing and image reconstruction, Inverse Problems, 20 (2004), 103-120. 4

[9] G. H. G. Chen, R. T. Rockafellar, Convergence rates in forward-backward splitting, SIAM J. Optim., 7 (1997), 421-444. 1

[10] C. Chidume, Geometric properties of Banach spaces and nonlinear iterations, Lecture Notes in Mathematics, SpringerVerlag London, Ltd., London, (2009). 2.2

[11] S. Y. Cho, B. A. Bin Dehaish, X.-L. Qin, Weak convergence of a splitting algorithm in Hilbert spaces, J. Appl. Anal. Comput., 7 (2017), 427-438. 1

[12] P. Cholamjiak, A generalized forward-backward splitting method for solving quasi inclusion problems in Banach spaces, Numer. Algorithms, 2015 (2015), 915-932. 1, 2.7

[13] I. Cioranescu, Geometry of Banach spaces, duality mappings and nonlinear problems, Mathematics and its Applications, Kluwer Academic Publishers Group, Dordrecht, (1990). 2.1

[14] P. L. Combettes, Iterative construction of the resolvent of a sum of maximal monotone operators, J. Convex Anal., 16 (2009), 727-748. 1

[15] P. L. Combettes, V. R. Wajs, Signal recovery by proximal forward-backward splitting, Multiscale Model. Simul., 4 (2005), 1168-1200. 1

[16] P. Deuhard, Recent progress in extrapolation methods for ordinary differential equations, SIAM Rev., 27 (1985), 505-535. 1

[17] J. Douglas, Jr., H. H. Rachford, Jr., On the numerical solution of heat conduction problems in two and three space variables, Trans. Amer. Math. Soc., 82 (1956), 421-439. 1

[18] J. C. Dunn, Convexity, monotonicity, and gradient processes in Hilbert space, J. Math. Anal. Appl., 53 (1976), 145-158. 1

[19] O. Güler, On the convergence of the proximal point algorithm for convex minimization, SIAM J. Control Optim., 29 (1991), 403-419. 1

[20] S.-N. He, C.-P. Yang, Solving the variational inequality problem defined on intersection of finite level sets, Abstr. Appl. Anal., 2013 (2013), 8 pages. 2.5

[21] P.-L. Lions, B. Mercier, Splitting algorithms for the sum of two nonlinear operators, SIAM J. Numer. Anal., 16 (1979), 964-979. 1

[22] G. López, V. Martín-Márquez, F.-H. Wang, H.-K. Xu, Forward-backward splitting methods for accretive operators in Banach spaces, Abstr. Appl. Anal., 2012 (2012), 25 pages. 1, 1, 2.8, 2.9

[23] P. E. Maingé, Approximation methods for common fixed points of nonexpansive mappings in Hilbert spaces, J. Math. Anal. Appl., 325 (2007), 469-479. 2.4

[24] B. Martinet, Régularisation d'inéquations variationnelles par approximations successives, (French) Rev. Franaise Informat. Recherche Opérationnelle, 4 (1970), 154-158. 1

[25] D. S. Mitrinović, Analytic inequalities, In cooperation with P. M. Vasić, Die Grundlehren der mathematischen Wissenschaften, Band 165 Springer-Verlag, New York-Berlin, (1970). 2.6

[26] A. Moudafi, Viscosity approximation methods for fixed-points problems, J. Math. Anal. Appl., 241 (2000), 46-55. 1

[27] G. B. Passty, Ergodic convergence to a zero of the sum of monotone operators in Hilbert space, J. Math. Anal. Appl., 72 (1979), 383-390. 1

[28] D. H. Peaceman, H. H. Rachford, Jr., The numerical solution of parabolic and elliptic differential equations, J. Soc. Indust. Appl. Math., 3 (1955), 28-41. 1

[29] X.-L. Qin, S. Y. Cho, L. Wang, A regularization method for treating zero points of the sum of two monotone operators, Fixed Point Theory Appl., 2014 (2014), 10 pages. 1

[30] X.-L. Qin, J.-C. Yao, Weak convergence of a Mann-like algorithm for nonexpansive and accretive operators, J. Inequal. Appl., 2016 (2016), 9 pages. 1

[31] S. Reich, Strong convergence theorems for resolvents of accretive operators in Banach spaces, J. Math. Anal. Appl., 75 (1980), 287-292. 2.3

[32] R. T. Rockafellar, On the maximal monotonicity of subdifferential mappings, Pacific J. Math., 33 (1970), 209-216. 4

[33] R. T. Rockafellar, Monotone operators and the proximal point algorithm, SIAM J. Control. Optim., 14 (1976), 877-898. 1

[34] C. Schneider, Analysis of the linearly implicit mid-point rule for differential-algebraic equations, Electron. Trans. Numer. Anal., 1 (1993), 1-10. 1

[35] S. Somali, Implicit midpoint rule to the nonlinear degenerate boundary value problems, Int. J. Comput. Math., 79 (2002), 327-332. 1 
[36] W. Takahashi, N.-C. Wong, J.-C. Yao, Two generalized strong convergence theorems of Halpern's type in Hilbert spaces and applications, Taiwanese J. Math., 16 (2012), 1151-1172. 1, 1

[37] P. Tseng, A modified forward-backward splitting method for maximal monotone mappings, SIAM J. Control Optim., 38 (2000), 431-446. 1, 1

[38] M. van Veldhuizen, Asymptotic expansions of the global error for the implicit midpoint rule (stiff case), Computing, 33 (1984), 185-192. 1

[39] F.-H. Wang, H.-H. Cui, On the contraction-proximal point algorithms with multi-parameters, J. Global Optim., 54 (2012), 485-491. 1

[40] H.-K. Xu, Inequalities in Banach spaces with applications, Nonlinear Anal., 16 (1991), 1127-1138. 2

[41] H.-K. Xu, Viscosity approximation methods for nonexpansive mappings, J. Math. Anal. Appl., 298 (2004), 279-291. 1

[42] H.-T. Zegeye, N. Shahzad, Strong convergence theorems for a common zero for a finite family of m-accretive mappings, Nonlinear Anal., 66 (2007), 1161-1169. 1 